\title{
Basal cell carcinoma of the nipple: a rare case report
}

\author{
DrKoushik Dewan ${ }^{1}$, DrPrabir Kumar Jash ${ }^{2}$, DrDebarati Chattopadhyay ${ }^{3}$, \\ DrAbhishek Saraf ${ }^{4}$,Dr Sunil Np ${ }^{5}$, DrSouradip Gupta ${ }^{6}$ \\ 1.Demonstrator,Department of Laboratory Medicine, School of Tropical Medicine,Kolkata-73,India \\ 2. Associate Professor,Department of Plastic Surgery, Medical College Kolkata, Kolkata-73,India \\ 3. R.M.O cum Clinical Tutor, Department of Plastic Surgery, IPGME\&R, Kolkata-20,India \\ 4,5,6-Resident, Department of Plastic Surgery, Medical College Kolkata, Kolkata-73,India
}

\begin{abstract}
Although Basal cell carcinoma is the most common skin malignancy, it is extremely rare in covered parts of the body like the nipple-areola complex in female. Very few cases have been reported in English literature till date. The present case adds to the few reported cases of BCC over nipple areolar complex and thus highlights the importance of identifying BCC as a potential diagnosis in any ulcer over the nipple areola complex.
\end{abstract}

Keywords:Basal cell carcinoma(BCC),Nipple areolar complex.

\section{Introduction}

Although Basal cell carcinoma (BCC) is the most common malignancy of the skin, BCC occurring on the nipple-areola complex (NAC) is extremely rare. Since its first description by Robinson in $1893^{1}$, there are only 39 reported cases of BCCs of the NAC in the English literature ${ }^{2,3}$. Of these 25 are male and only 14 are female. Here we describe a case of basal call carcinoma of the nipple in a 36 year female.

\section{Case Report}

A 36-year lady presented with the history of a hyperpigmented mass over her left nipple areola complex for the past 3 years. It had gradually enlarged over the said period. The mass was itchy. There was no history of nipple retraction or discharge. There was no similar family history. There was no history of exposure to radiation or arsenic exposure. On examination a single hyper pigmented ulcerated mass $4 \mathrm{~cm} \times 5 \mathrm{~cm}$ in size was found involving the left nipple areola complex. [Figure 1]. No axillary lymph node enlargement was detected clinically. Mammography did not detect any lesion within the breast parenchyma of either side. Fine needle aspiration cytology of the lesion suggested Basal cell carcinoma. Wide local excision of the mass was done. The histopathological study showed basaloid cell with high $\mathrm{N}: \mathrm{C}$ in nests with peripheral palisading and adenoid cystic pattern with focal involvement of lactiferous duct. All resection margins were free without any features of Paget's disease of nipple or ductal carcinoma breast.[Figure 2]. The patient is doing well on follow up, with no sign of local recurrence or distant metastasis at 8 months after tumor excision.

\section{Discussion}

Non melanoma skin cancers are the most common dermatological malignancies, with Basal cell carcinomas accounting for 70-80\% of them. ${ }^{4}$ BCCs are most commonly found in the sun-exposed areas of the head and neck. As the nipple areola region is usually not exposed to sunlight, the occurrence of BCC in this site is quite rare with a male predominance in the reported cases. 39 cases of $\mathrm{BCC}$ of the nipple areola complex have been described in literature in the last 121 years of which only 14 are female. The male preponderance is probably due to the increased exposure of the male chest to sunlight.

The differential diagnosis of BCC of nipple areola complex include Paget's disease, malignant melanoma, Bowen's disease and contact dermatitis. ${ }^{5}$

$\mathrm{BCC}$ has low metastatic potential. The overall rate of metastasis in BCC ranges from 0.0028 to $0.5 \%{ }^{6}$ However BCCs located on the NAC have been considered to be more aggressive with a metastatic rate of $9.1 \%$ $11.5 \% .{ }^{2,7}$ The higher propensity of metastasis in this location is possibly due to the rich network of lymphatic capillaries in the subareolar plexus that provides an easy route for tumor spread.

The management of BCC of nipple areolar complex is thus slightly different from that at other sites. In addition to photodynamic therapy, lasers, Mohs' microsurgery, simple excision with or without radiotherapy, partial mastectomy with axillary dissection have also been described, given the higher propensity of metastasis. Moreover the role of sentinel lymph node biopsy as a part of the surgical protocol is being considered. ${ }^{2}$ 


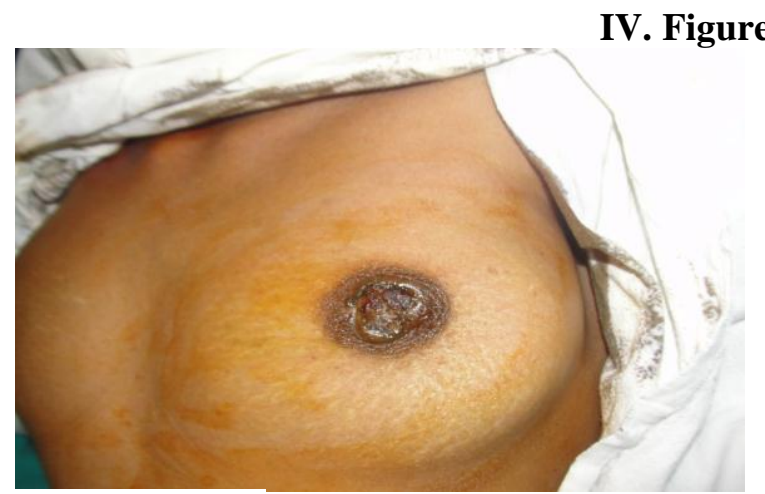

FIGURE1:- Ulcerated mass over left nipple areola complex

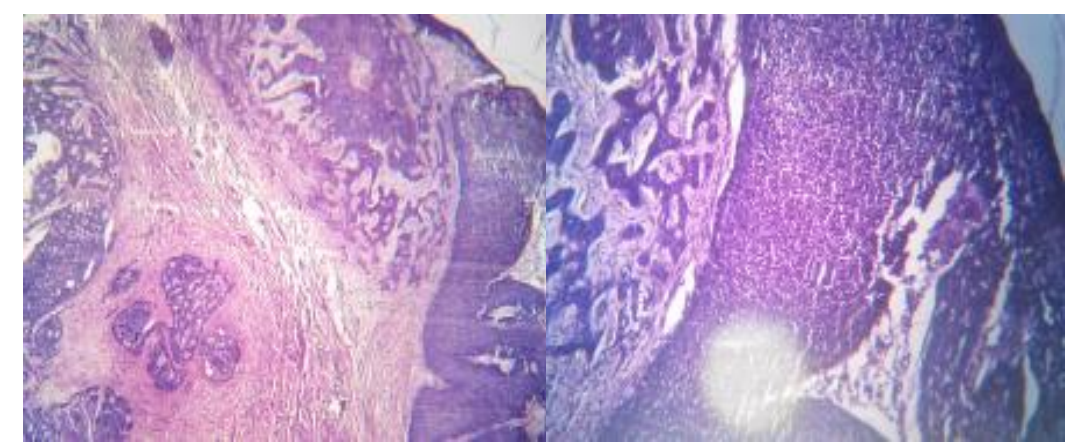

\section{A(H\&E) 100X \\ B(H\&E)400X \\ FIGURE2A\&B-Microphotographs showing features of basal cell carcinoma.}

\section{Conclusion}

BCC of the nipple areola complex is rare and more so in the female. The present case adds to the few reported cases of BCC over nipple areola complex and thus highlights the importance of identifying BCC as a potential diagnosis in any ulcer over the nipple areola complex.

\section{References}

[1]. Robinson HB.Rodent ulcer of the male breast. Trans PatholSocLond 1893;44:147-8

[2]. Takeno S, Kikuchi N, Miura T, Anami K, Takahashi Y, Hashimoto T, et al. Basal cell carcinoma of the nipple in male patients with gastriccancer recurrence: report of a case. Breast Cancer 2014; 21:102-107

[3]. Oram Y, Demirkesen C, Akkaya AD, KoyuncuE.Basal Cell Carcinoma of the Nipple: An Uncommon but Ever-Increasing Location. Case Rep Dermatol Med 2011;2011:818291

[4]. Kwasniak LA, Garcia-Zuazaga J. Basal cell carcinoma: evidence-based medicine and review of treatment modalities. Int J Dermatol 2011:50:645-658

[5]. Benharroch D, Geffen DB, Peiser J, Rosenberg L. Basal cell carcinoma of the male nipple: case report and review of the literature. J DermatolSurgOncol 1993;19:137-139

[6]. Sinha A, Langtry JA. Secondary intention healing following Mohs micrographic surgery for basal cell carcinoma of the nipple and areola. ActaDermVenereol. 2010;91:78-9

[7]. Ferguson MS, Nouraei SAR, Davies BJH, McLean NR. Basal cell carcinoma of the nipple-areola complex. DermatolSurg 2009;35: 1771-177 